TRANSACTIONS OF THE

AMERICAN MATHEMATICAL SOCIETY

Volume 356, Number 2, Pages 599-620

S 0002-9947(03)03104-0

Article electronically published on September 22, 2003

\title{
THE DISTRIBUTION OF PRIME IDEALS OF IMAGINARY QUADRATIC FIELDS
}

\author{
G. HARMAN, A. KUMCHEV, AND P. A. LEWIS
}

\begin{abstract}
Let $Q(x, y)$ be a primitive positive definite quadratic form with integer coefficients. Then, for all $(s, t) \in \mathbb{R}^{2}$ there exist $(m, n) \in \mathbb{Z}^{2}$ such that $Q(m, n)$ is prime and

$$
Q(m-s, n-t) \ll Q(s, t)^{0.53}+1 .
$$
\end{abstract}

This is deduced from another result giving an estimate for the number of prime ideals in an ideal class of an imaginary quadratic number field that fall in a given sector and whose norm lies in a short interval.

\section{INTRODUCTION}

Let $K=\mathbb{Q}(\sqrt{d})$ be an imaginary quadratic field of discriminant $d<0$, and let $\mathfrak{O}=\mathfrak{O}_{K}$ be its ring of integers. In this paper, we study the distribution of the prime ideals of $\mathfrak{O}$ in small regions of a certain type. Our main result is Theorem 2 below, which improves on earlier work by Coleman (see 4, Theorem 2.1] and [7. Theorem 4]). When combined with [4, Proposition 2.3], Theorem 2 yields the following result.

Theorem 1. Let $d<0$ be the discriminant of an imaginary quadratic field $K$, and let $Q(x, y) \in \mathbb{Z}[x, y]$ be a positive definite quadratic form with discriminant $d$. Then, for every pair $(s, t) \in \mathbb{R}^{2}$, there is another pair $(m, n) \in \mathbb{Z}^{2}$ for which $Q(m, n)$ is prime and

$$
Q(s-m, t-n) \ll Q(s, t)^{0.53}+1 .
$$

The implied constant depends only on $K$.

In the special case when $Q(x, y)=x^{2}+y^{2}$, we get the following

Corollary. For every $z \in \mathbb{C}$, one can find a Gaussian prime $\pi \neq z$ satisfying

$$
|z-\pi| \ll|z|^{0.53}+1 \text {. }
$$

If we take $(s, t) \in \mathbb{Z}^{2}$ so that $Q(s, t)$ is a prime, Theorem 1 provides a bound on the gaps between points at which the form $Q(x, y)$ attains prime values. Therefore, one may compare the above result with the estimates for the difference between consecutive primes. In such a comparison, the exponent of $Q(s, t)$ on the right side of (1.1) corresponds to the smallest $\theta$ such that, for sufficiently large values of $x$,

Received by the editors January 11, 2002 and, in revised form, April 22, 2002.

2000 Mathematics Subject Classification. Primary 11R44; Secondary 11N32, 11N36, 11 N42.

The second author was partially supported by NSF Grant DMS 9970455 and NSERC Grant A5123.

The third author was supported by an EPSRC Research Studentship. 
the interval $\left(x-x^{\theta}, x\right]$ always contains a prime number. Here is how Theorem 1 and recent work preceding it compare with the respective results concerning primes in short intervals:

- the exponent $7 / 12+\varepsilon$ obtained by Coleman [4, Theorem 1.1] corresponds to a celebrated result by Huxley [13] (henceforth, $\varepsilon$ denotes a sufficiently small, positive constant, not necessarily the same in each occurrence);

- the exponent 11/20 that follows from [7, Theorem 4] corresponds to the main result of Heath-Brown and Iwaniec [12];

- Theorem 11 corresponds to the existence of rational primes in all intervals $\left(x-x^{0.53}, x\right]$ with $x \geq x_{0}$, and compares with recent results by Baker and Harman [1] and by Baker, Harman and Pintz [3] in which the lengths of the intervals are $x^{0.535}$ and $x^{0.525}$, respectively.

Our method is an adaptation to algebraic number fields of the sieve method introduced by the first author [8, 9] and shares many features with [2] and [3]. Our result is not quite as strong as the result in 3] because we do not have an analogue for Hecke $L$-functions of Watt's mean-value theorem [18].

In order to state the main result, we need to establish some notation. We denote by $\mathfrak{a}, \mathfrak{b}, \ldots$ the integral ideals of $\mathfrak{O}$ and reserve $\mathfrak{p}$ (with or without subscripts) for prime ideals; also, we write $\hat{\alpha}, \hat{\beta}, \ldots$ for the ideal numbers of $K$ and $\hat{\pi}$ for the prime ideal numbers.

Let $w$ be the number of roots of unity contained in the field $K$. The Hecke Grössencharaktere $\lambda(\cdot)$ is defined on the multiplicative group of the ideal numbers of $K$ by

$$
\lambda(\hat{\mu})=\left(\frac{\hat{\mu}}{|\hat{\mu}|}\right)^{w} .
$$

If $\mathcal{C}$ is an ideal class, let $\mathcal{C}^{-1}$ denote its inverse in the ideal class group. For each class $\mathcal{C}$, we choose and fix an ideal $\mathfrak{a}_{0} \in \mathcal{C}^{-1}$. Then, given an ideal $\mathfrak{a} \in \mathcal{C}$, we can find an algebraic integer $\xi_{\mathfrak{a}} \in \mathfrak{a}_{0}$ with $\left(\xi_{\mathfrak{a}}\right)=\mathfrak{a} \mathfrak{a}_{0}$. Moreover, $\xi_{\mathfrak{a}}$ is unique up to multiplication by units, whence $\arg \lambda\left(\xi_{\mathfrak{a}}\right)$ is unique $\bmod 2 \pi$. Therefore, if $\mathcal{C}$ is an ideal class and $x, y, \phi_{0}, \phi$ are real numbers subject to the restrictions $0<\phi<2 \pi$ and $0<y<x$, the following set of ideals is well-defined:

$$
\mathcal{A}=\left\{\mathfrak{a} \in \mathcal{C}: x-y \leq N \mathfrak{a}<x, \phi_{0} \leq \arg \lambda\left(\xi_{\mathfrak{a}}\right) \leq \phi_{0}+\phi\right\} .
$$

Our main theorem can now be stated as follows.

Theorem 2. There is an $x_{0}>0$ such that if $x \geq x_{0}, y \geq x^{0.765}, \phi \geq x^{-0.235}$, and $\mathcal{A}$ is the collection of ideals defined by (1.2), then

$$
\sum_{\mathfrak{p} \in \mathcal{A}} 1 \gg \frac{\phi y}{\log x} .
$$

One expects that one should actually be able to prove an asymptotic formula for the left-hand side of (1.3) whenever $y \gg x^{1 / 2+\varepsilon}$ and $\phi \gg x^{-1 / 2+\varepsilon}$. However, the exponents $19 / 24+\varepsilon$ and $-5 / 24+\varepsilon$ obtained in [4] seem to be the best the present methods can produce if one insists on having an asymptotic formula. In fact, even assuming the Riemann hypothesis for the Dedekind zeta function $\zeta_{K}(s)$, we do not know how to get exponents better than $3 / 4+\varepsilon$ and $-1 / 4+\varepsilon$, respectively. The related problem where one only restricts $\arg \lambda\left(\xi_{\mathfrak{a}}\right)$ and not $N \mathfrak{a}$ is studied by the first and third authors elsewhere [10]. 


\section{Outline of the method}

We consider a set $\mathcal{A}$ defined by (1.2) with $y \geq x^{(\theta+1) / 2+\varepsilon}$ and $\phi \geq x^{(\theta-1) / 2+\varepsilon}$, and aim to prove (1.3) when $\theta \geq 0.53-2 \varepsilon$. Let $y_{1}=x \exp \left(-3 \log ^{1 / 3} x\right)$ and let $\mathcal{C}$ be the ideal class appearing in the definition of the set $\mathcal{A}$. We define

$$
\mathcal{B}=\left\{\mathfrak{a} \in \mathcal{C}: x-y_{1} \leq N \mathfrak{a}<x\right\} .
$$

In order to simplify the proof, we will replace the sum on the left side of (1.3) by $\sum_{\mathfrak{p}} \Psi(\mathfrak{p})$, where $\Psi(\cdot)$ is a smooth function that approximates the characteristic function of $\mathcal{A}$ from below (see (3.5) for the precise definition of $\Psi$ ). Clearly, it suffices to show that

$$
\sum_{\mathfrak{p}} \Psi(\mathfrak{p}) \gg \frac{\phi y}{\log x}
$$

If $z \geq 2$, we define the quantities

$$
S(\mathcal{A}, z)=\sum_{(\mathfrak{a}, \mathfrak{P}(z))=1} \Psi(\mathfrak{a}) \quad \text { and } \quad S(\mathcal{B}, z)=\sum_{\substack{\mathfrak{a} \in \mathcal{B} \\(\mathfrak{a}, \mathfrak{P}(z))=1}} 1,
$$

where $\mathfrak{P}(z)$ is the ideal

$$
\mathfrak{P}(z)=\prod_{N \mathfrak{p}<z} \mathfrak{p} .
$$

The left-hand side of $(2.2)$ can then be expressed as $S\left(\mathcal{A}, x^{1 / 2}\right)$.

Our programme is as follows. First, we represent $S\left(\mathcal{A}, x^{1 / 2}\right)$ as a linear combination of sifting sums that are easier to handle, say

$$
S\left(\mathcal{A}, x^{1 / 2}\right)=\sum_{j=1}^{k} S_{j}-\sum_{j=k+1}^{\ell} S_{j} .
$$

Here, $S_{j} \geq 0$ and for $j \leq k^{\prime}<k$ and $j>k$, we will be able to prove asymptotic formulae of the form

$$
S_{j}=\frac{\phi y}{2 \pi y_{1}} S_{j}^{*}(1+o(1))
$$

where $S_{j}^{*}$ is a sifting function analogous to $S_{j}$ in which the set $\mathcal{B}$ is being sifted instead. We then decompose $S\left(\mathcal{B}, x^{1 / 2}\right)$ following the steps that led us to (2.3) and combine the two decompositions by means of the asymptotic formulae (2.4). Discarding the sums $S_{j}, k^{\prime}<j \leq k$, we now get the lower bound

$$
S\left(\mathcal{A}, x^{1 / 2}\right) \geq \frac{\phi y}{2 \pi y_{1}}\left(S\left(\mathcal{B}, x^{1 / 2}\right)-\sum_{k^{\prime}<j \leq k} S_{j}^{*}\right)(1+o(1)) .
$$

It remains to ensure that the right-hand side of this inequality is nonnegative, that is, that not too many sums $S_{j}$ are discarded.

The plan of the paper is as follows. In Section 3, we establish a connection between mean-value estimates for Dirichlet polynomials and asymptotic formulae of the form

$$
\sum_{\mathfrak{a}} c(\mathfrak{a}) \Psi(\mathfrak{a})=\frac{\phi y}{2 \pi y_{1}} \sum_{\mathfrak{a} \in \mathcal{B}} c(\mathfrak{a})+\text { error terms. }
$$

Section 4 contains auxiliary results about Dirichlet polynomials. In Section 5 , we combine the results from Sections 3 and 4 , and prove asymptotic formulae of the 
form (2.4) for a variety of sifting functions $S_{j}$. Finally, in Section 6 , we construct the actual decomposition (2.3) and show that the resulting lower bound is indeed positive.

Notation. We shall write $N \mathfrak{a} \sim X$ for the condition $X \leq N \mathfrak{a}<2 X$ and $N \mathfrak{a} \asymp X$ for the condition $c_{1} X \leq N \mathfrak{a}<c_{2} X$, where $c_{1}$ and $c_{2}$ are some absolute constants. We shall use the letter $B$ to denote a sufficiently large, positive constant, not necessarily the same in each occurrence; $\eta$ will stand for a fixed positive number, sufficiently small in terms of $\varepsilon$. We shall use the following arithmetic functions:

$\tau(\mathfrak{a})$ : the number of ideals dividing the ideal $\mathfrak{a}$;

$\mu(\mathfrak{a})$ : the analogue for ideals of the Möbius function:

$$
\mu(\mathfrak{a})= \begin{cases}1 & \text { if } \mathfrak{a}=\mathfrak{O} \\ (-1)^{k} & \text { if } \mathfrak{a} \text { is the product of } k \text { distinct prime ideals } \\ 0 & \text { if } \mathfrak{p}^{2} \mid \mathfrak{a} \text { for some } \mathfrak{p}\end{cases}
$$

$\rho(\mathfrak{a}, z)$ : for real $z \geq 2$, we define

$$
\rho(\mathfrak{a}, z)= \begin{cases}1 & \text { if }(\mathfrak{a}, \mathfrak{P}(z))=1 \\ 0 & \text { otherwise }\end{cases}
$$

\section{Reduction OF THE PROBLEM}

In this section we demonstrate that asymptotic formulae of the form 2.5 can be derived from mean-value estimates for Dirichlet polynomials

$$
F\left(s, \lambda^{m}\right)=\sum_{\mathfrak{a}} c(\mathfrak{a}) \lambda^{m}(\mathfrak{a})(N \mathfrak{a})^{-s}
$$

where $\lambda$ is the Hecke Grössencharaktere defined above, the summation is over integral ideals $\mathfrak{a} \in \mathcal{C}$ with $N \mathfrak{a} \asymp x$, and the coefficients $c(\mathfrak{a})$ satisfy

$$
|c(\mathfrak{a})| \leq \tau(\mathfrak{a})^{B}
$$

Sometimes, when $m=0$ in (3.1), we will write simply $F(s)$.

We start by defining the function $\Psi(\mathfrak{a})$. Let $\Delta_{1}=y x^{-\eta}$, and let $\psi_{1}(t)$ be a function of the $C^{\infty}(\mathbb{R})$ class satisfying:

- $\psi_{1}(t)=1$ if $x-y+\Delta_{1} \leq t \leq x-\Delta_{1}$;

- $\psi_{1}(t)=0$ if $t \notin(x-y, x)$;

- $0 \leq \psi_{1}(t) \leq 1$ if $x-y \leq t \leq x$;

- $\psi_{1}^{(j)}(t) \ll j^{2 j} \Delta_{1}^{-j}$ for $j=1,2, \ldots$.

Also, let $\Delta_{2}=\phi x^{-\eta}$, let $r=[2 / \eta]+1$, and let $\psi_{2}(t)$ be a $2 \pi$-periodic function satisfying:

- $\psi_{2}(t)=1$ if $\phi_{0}+\Delta_{2} \leq t \leq \phi_{0}+\phi-\Delta_{2}$;

- $\psi_{2}(t)=0$ if $\phi_{0}+\phi \leq t \leq \phi_{0}+2 \pi$;

- $0 \leq \psi_{2}(t) \leq 1$ if $\phi_{0} \leq t \leq \phi_{0}+\phi$;

- $\psi_{2}(t)$ has the Fourier expansion

$$
\psi_{2}(t)=\widehat{\psi}_{2}(0)+\sum_{m \neq 0} \widehat{\psi}_{2}(m) e^{i m t},
$$


where $\widehat{\psi}_{2}(0)=\left(\phi-\Delta_{2}\right) / 2 \pi$ and, for $m \neq 0$,

$$
\left|\widehat{\psi}_{2}(m)\right| \leq \min \left(\phi, \frac{2}{\pi|m|}, \frac{2}{\pi|m|}\left(\frac{2 r}{|m| \Delta_{2}}\right)^{r}\right) .
$$

We now define $\Psi(\mathfrak{a})$ by

$$
\Psi(\mathfrak{a})= \begin{cases}\psi_{1}(N \mathfrak{a}) \psi_{2}\left(\arg \lambda\left(\xi_{\mathfrak{a}}\right)\right), & \mathfrak{a} \in \mathcal{C}, \\ 0, & \mathfrak{a} \notin \mathcal{C} .\end{cases}
$$

Let us consider the left-hand side of (2.5). From (3.3) and the definition of $\xi_{\mathfrak{a}}$, we get

$$
\psi_{2}\left(\arg \lambda\left(\xi_{\mathfrak{a}}\right)\right)=\sum_{m \in \mathbb{Z}} \widehat{\psi}_{2}(m) \lambda^{m}\left(\mathfrak{a a}_{0}\right)
$$

Thus, choosing $M=\left[\Delta_{2}^{-1} x^{\eta}\right]+1$, and using (3.4) to estimate the tail of the last series, we obtain

$$
\psi_{2}\left(\arg \lambda\left(\xi_{\mathfrak{a}}\right)\right)=\sum_{|m| \leq M} \widehat{\psi}_{2}(m) \lambda^{m}\left(\mathfrak{a a}_{0}\right)+O\left(x^{-2}\right) .
$$

Hence,

$$
\sum_{\mathfrak{a}} c(\mathfrak{a}) \Psi(\mathfrak{a})=\sum_{|m| \leq M} \widehat{\psi}_{2}(m) \lambda^{m}\left(\mathfrak{a}_{0}\right) \sum_{\mathfrak{a} \in \mathcal{C}} c(\mathfrak{a}) \lambda^{m}(\mathfrak{a}) \psi_{1}(N \mathfrak{a})+O(1) .
$$

Let

$$
\widehat{\psi}_{1}(s)=\int_{0}^{\infty} \psi_{1}(t) t^{s-1} d t
$$

be the Mellin transform of $\psi_{1}(t)$. By Mellin's inversion formula, we have

$$
\psi_{1}(t)=\frac{1}{2 \pi i} \int_{\frac{1}{2}-i \infty}^{\frac{1}{2}+i \infty} \widehat{\psi}_{1}(s) t^{-s} d s .
$$

Thus, upon noting that $\psi_{1}(N \mathfrak{a})=0$ unless $N \mathfrak{a} \asymp x$, we get

$$
\sum_{\mathfrak{a}} c(\mathfrak{a}) \Psi(\mathfrak{a})=\frac{1}{2 \pi i} \sum_{|m| \leq M} \widehat{\psi}_{2}(m) \lambda^{m}\left(\mathfrak{a}_{0}\right) \int_{\frac{1}{2}-i \infty}^{\frac{1}{2}+i \infty} F\left(s, \lambda^{m}\right) \widehat{\psi}_{1}(s) d s+O(1),
$$

where $F\left(s, \lambda^{m}\right)$ is the Dirichlet polynomial (3.1). Now, we observe that $r$ partial integrations yield the bound

$$
\widehat{\psi}_{1}(\sigma+i t) \ll \Delta_{1} x^{\sigma-1}\left(\frac{x r^{2}}{|t| \Delta_{1}}\right)^{r} \quad(r=1,2, \ldots) .
$$

Applying this estimate with $r=[2 / \eta]+1$, we see that

$$
\sum_{\mathfrak{a}} c(\mathfrak{a}) \Psi(\mathfrak{a})=\frac{1}{2 \pi i} \sum_{|m| \leq M} \widehat{\psi}_{2}(m) \lambda^{m}\left(\mathfrak{a}_{0}\right) \int_{\frac{1}{2}-i T_{1}}^{\frac{1}{2}+i T_{1}} F\left(s, \lambda^{m}\right) \widehat{\psi}_{1}(s) d s+O(1),
$$

where $T_{1}=x^{1+\eta} \Delta_{1}^{-1}$.

Let us now suppose that the following mean-value bounds hold for $F\left(s, \lambda^{m}\right)$ :

$$
\sum_{0<|m| \leq M} \int_{-T_{1}}^{T_{1}}\left|F\left(\frac{1}{2}+i t, \lambda^{m}\right)\right| d t \ll x^{1 / 2} \exp \left(-B(\log x)^{1 / 4}\right),
$$


and

$$
\int_{T_{0}}^{T_{1}}\left|F\left(\frac{1}{2}+i t, \lambda^{0}\right)\right| d t \ll x^{1 / 2} \exp \left(-B(\log x)^{1 / 4}\right),
$$

where in (3.8), $T_{0}=\exp \left(\log ^{1 / 3} x\right)$. Since

$$
\left|\widehat{\psi}_{1}(\sigma+i t)\right| \leq y x^{\sigma-1} \quad \text { and } \quad\left|\widehat{\psi}_{2}(m)\right| \leq \phi,
$$

we infer from (3.6) $-(3.8)$ that

$$
\sum_{\mathfrak{a}} c(\mathfrak{a}) \Psi(\mathfrak{a})=\frac{\widehat{\psi}_{2}(0)}{2 \pi i} \int_{\frac{1}{2}-i T_{0}}^{\frac{1}{2}+i T_{0}} F(s) \widehat{\psi}_{1}(s) d s+O\left(\phi y \exp \left(-B(\log x)^{1 / 4}\right)\right) .
$$

Furthermore, we have

$$
\widehat{\psi}_{1}(s)=\widehat{\psi}_{1}(1) x^{s-1}+O\left(|s| y^{2} x^{\sigma-2}\right)
$$

and $F\left(\frac{1}{2}+i t\right) \ll x^{1 / 2}(\log x)^{B} ;$ so (3.9) yields

$$
\sum_{\mathfrak{a}} c(\mathfrak{a}) \Psi(\mathfrak{a})=\frac{\phi y}{4 \pi^{2} i} \int_{\frac{1}{2}-i T_{0}}^{\frac{1}{2}+i T_{0}} F(s) x^{s-1} d s+O\left(\phi y \exp \left(-B(\log x)^{1 / 4}\right)\right) .
$$

Now, let $y_{1}=x \exp \left(-3 \log ^{1 / 3} x\right)$, let $\Delta_{3}=y_{1} x^{-\eta}$, and let $\psi_{3}(t)$ be a function analogous to $\psi_{1}(t)$ with $y_{1}$ and $\Delta_{3}$ in place of $y$ and $\Delta_{1}$. Then, by a simpler version of the above argument, we obtain

$$
\sum_{\mathfrak{a} \in \mathcal{C}} c(\mathfrak{a}) \psi_{3}(N \mathfrak{a})=\frac{y_{1}}{2 \pi i} \int_{\frac{1}{2}-i T_{0}}^{\frac{1}{2}+i T_{0}} F(s) x^{s-1} d s+O\left(y_{1} \exp \left(-B(\log x)^{1 / 4}\right)\right),
$$

provided that (3.8) holds. On the other hand,

$$
\sum_{\mathfrak{a} \in \mathcal{C}} c(\mathfrak{a}) \psi_{3}(N \mathfrak{a})=\sum_{\mathfrak{a} \in \mathcal{B}} c(\mathfrak{a})+O\left(x^{1-\eta}\right)
$$

so we deduce that

$$
\sum_{\mathfrak{a} \in \mathcal{B}} c(\mathfrak{a})=\frac{y_{1}}{2 \pi i} \int_{\frac{1}{2}-i T_{0}}^{\frac{1}{2}+i T_{0}} F(s) x^{s-1} d s+O\left(y_{1} \exp \left(-B(\log x)^{1 / 4}\right)\right) .
$$

Upon combining (3.10) and (3.11), we have now completed the proof of the following result.

Lemma 1. Let the Dirichlet polynomial $F\left(s, \lambda^{m}\right)$ be defined by (3.1), and suppose that its coefficients satisfy (3.2). Also, suppose that inequalities (3.7) and (3.8) hold. Then

$$
\sum_{\mathfrak{a}} c(\mathfrak{a}) \Psi(\mathfrak{a})=\frac{\phi y}{2 \pi y_{1}} \sum_{\mathfrak{a} \in \mathcal{B}} c(\mathfrak{a})+O\left(\phi y \exp \left(-B(\log x)^{1 / 4}\right)\right) .
$$

Lemma 1 will be our primary tool for proving asymptotic formulae of the form (2.5), but there is one case in which we will not be able to satisfy its hypotheses and will need an alternative device. The next lemma provides such a device.

Lemma 2. Define the Dirichlet polynomial

$$
F\left(s, \lambda^{m}\right)=\sum_{\mathfrak{a}, \mathfrak{b}} c(\mathfrak{a}) \lambda^{m}(\mathfrak{a} \mathfrak{b}) N(\mathfrak{a} \mathfrak{b})^{-s},
$$


where the coefficients $c(\mathfrak{a})$ satisfy (3.2) and the summation is over integral ideals $\mathfrak{a}$ and $\mathfrak{b}$ subject to

$$
\mathfrak{a} \mathfrak{b} \in \mathcal{C}, \quad N \mathfrak{a} \leq x^{\eta}, \quad N(\mathfrak{a} \mathfrak{b}) \asymp x
$$

If (3.7) holds, then

$$
\sum_{\substack{\mathfrak{a}, \mathfrak{b}: \\ N \mathfrak{a} \leq x^{\eta}}} c(\mathfrak{a}) \Psi(\mathfrak{a} \mathfrak{b})=\frac{\phi y}{2 \pi y_{1}} \sum_{\substack{\mathfrak{a} \mathfrak{b} \in \mathcal{B} \\ N \mathfrak{a} \leq x^{\eta}}} c(\mathfrak{a})+O\left(\phi y \exp \left(-B(\log x)^{1 / 4}\right)\right) .
$$

Proof. As in the proof of Lemma 1, we find that the left-hand side of (3.15) equals

$$
\sum_{|m| \leq M} \widehat{\psi}_{2}(m) \lambda^{m}\left(\mathfrak{a}_{0}\right) \sum_{\mathfrak{a}, \mathfrak{b}: \sqrt{3.14}} c(\mathfrak{a}) \lambda^{m}(\mathfrak{a} \mathfrak{b}) \psi_{1}(N(\mathfrak{a} \mathfrak{b}))+O(1),
$$

where $M=\left[\Delta_{2}^{-1} x^{\eta}\right]+1$. As before, we can estimate the contribution from $|m|>0$ by using the properties of the Mellin transform $\widehat{\psi}_{1}(s)$ and hypothesis (3.7). We obtain

$$
\begin{aligned}
\sum_{\mathfrak{a}, \mathfrak{b}:[3.14]} c(\mathfrak{a}) \Psi(\mathfrak{a} \mathfrak{b}) & =\widehat{\psi}_{2}(0) \sum_{\mathfrak{a}, \mathfrak{b}:[\sqrt[3.14]{3}} c(\mathfrak{a}) \psi_{1}(N(\mathfrak{a} \mathfrak{b})) \\
& +O\left(\phi y \exp \left(-B(\log x)^{1 / 4}\right)\right) .
\end{aligned}
$$

We will use the asymptotic formula (see [15, Corollary 1 on p. 417])

$$
\sum_{\substack{\mathfrak{a} \in \mathcal{C} \\ N \mathfrak{a} \leq X}} 1=C_{K} \cdot X+O\left(X^{2 / 3+\eta}\right),
$$

where $\mathcal{C}$ can be any ideal class and $C_{K}$ is a constant depending only on the field $K$ (and not on the class $\mathcal{C}$ ). From (3.16) and (3.17), we deduce

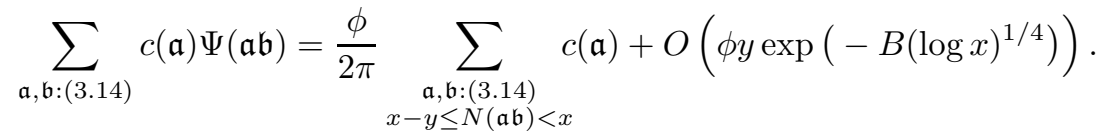

Let $\mathcal{C}_{1}, \ldots, \mathcal{C}_{h}$ be the distinct ideal classes of $K$. Then, using (3.17) again,

$$
\begin{aligned}
\sum_{\substack{\mathfrak{a}, \mathfrak{b}:(\sqrt[3]{3,14}) \\
x-y \leq N(\mathfrak{a} \mathfrak{b})<x}} c(\mathfrak{a}) & =\sum_{j=1}^{h} \sum_{\substack{\mathfrak{a} \in \mathcal{C}_{j} \\
N \mathfrak{a} \leq x^{\eta}}} c(\mathfrak{a}) \sum_{\substack{\mathfrak{b} \in \mathcal{C} \mathcal{C}_{j}^{-1} \\
x-y \leq N(\mathfrak{a} \mathfrak{b})<x}} 1 \\
& =C_{K} y \sum_{j=1}^{h} \sum_{\substack{\mathfrak{a} \in \mathcal{C}_{j} \\
N \mathfrak{a} \leq x^{\eta}}} \frac{c(\mathfrak{a})}{N \mathfrak{a}}+O\left(x^{2 / 3+2 \eta}\right) .
\end{aligned}
$$

Substituting this back into (3.18), we have

$$
\sum_{\substack{\mathfrak{a}, \mathfrak{b}: \\ N \mathfrak{a} \leq x^{\eta}}} c(\mathfrak{a}) \Psi(\mathfrak{a} \mathfrak{b})=C_{K} \cdot \frac{\phi y}{2 \pi} \sum_{j=1}^{h} \sum_{\substack{\mathfrak{a} \in \mathcal{C}_{j} \\ N \mathfrak{a} \leq x^{\eta}}} \frac{c(\mathfrak{a})}{N \mathfrak{a}}+O\left(\phi y \exp \left(-B(\log x)^{1 / 4}\right)\right) .
$$

The result follows from (3.20) and (3.19) with $y_{1}$ in place of $y$. 


\section{Dirichlet polynomials}

In this section we collect estimates for mean and large values of Dirichlet polynomials of the form (3.1). We will use these estimates to satisfy the hypotheses (3.7) and (3.8) of Lemma 1. We will always assume that the coefficients $c(\mathfrak{a})$ (if present) satisfy (3.2). Also, in this and often in the subsequent sections, we will use the same letter for a Dirichlet polynomial and for its length; for example, the summation in polynomial (3.1) will be over ideals with $N \mathfrak{a} \asymp F$.

4.1. Estimates for polynomials $F\left(s, \lambda^{m}\right)$. Let $2<T, M \leq x / 4$. If $\mathcal{S}$ is a set of pairs $(t, m)$, with $|t| \leq T,|m| \leq M$, we say that $\mathcal{S}$ is well-spaced if $\left|t-t^{\prime}\right| \geq 1$ whenever $(t, m),\left(t^{\prime}, m\right) \in \mathcal{S}$. We define the norm

$$
\|F\|_{p}= \begin{cases}\left(\sum_{(t, m) \in \mathcal{S}}\left|F\left(\frac{1}{2}+i t, \lambda^{m}\right)\right|^{p}\right)^{1 / p}, & 1 \leq p<\infty, \\ \sup _{(t, m) \in \mathcal{S}}\left|F\left(\frac{1}{2}+i t, \lambda^{m}\right)\right|, & p=\infty .\end{cases}
$$

Sometimes, we will need to impose the condition

$$
\|F\|_{\infty} \ll F^{1 / 2} \exp \left(-B(\log x)^{1 / 4}\right) .
$$

Lemma 3. Let $\mathcal{S}$ be well-spaced; then

$$
\|F\|_{2}^{2} \ll\left(M^{2}+T^{2}+F\right) G \log x,
$$

where

$$
G=G(F)=\sum_{N \mathfrak{a} \asymp F}|c(\mathfrak{a})|^{2}(N \mathfrak{a})^{-1} .
$$

Furthermore, if

$$
\left|F\left(\frac{1}{2}+i t, \lambda^{m}\right)\right| \geq V
$$

for all $(t, m) \in \mathcal{S}$, then the cardinality of $\mathcal{S}$ is bounded by

$$
|\mathcal{S}| \ll\left(\frac{G F}{V^{2}}+\frac{G^{3} F\left(M^{2}+T^{2}\right)}{V^{6}}\right) \log ^{4} x .
$$

Proof. The first assertion follows from [14 Lemma 1.4] and [4, Theorem 6.2]. The second assertion is [4, Lemma 7.3] with $\theta=0, \alpha(0)=1 / 2$, and $\beta(0)=2$.

The next two lemmas are analogues of [3, Lemma 9] and 2, Lemma 3]. In fact, if one replaces [2, Lemma 1] by Lemma 3] their proofs are almost identical to the proofs of those results.

Lemma 4. Let $P\left(s, \lambda^{m}\right), Q\left(s, \lambda^{m}\right), F\left(s, \lambda^{m}\right)$ be Dirichlet polynomials whose coefficients satisfy (3.2). Suppose that $P Q F=x$, and that $F\left(s, \lambda^{m}\right)$ satisfies (4.1). Let $\left(M^{2}+T^{2}\right) \leq x^{1-\theta-\eta}$, where $1 / 2+\eta<\theta \leq 7 / 12$, and let $g$ be a positive integer. Suppose that $P=x^{\sigma_{1}}, Q=x^{\sigma_{2}}$, with

$$
\begin{gathered}
\left|\sigma_{1}-\sigma_{2}\right| \leq 2 \theta-1, \\
1-\left(\sigma_{1}+\sigma_{2}\right) \leq \gamma(\theta),
\end{gathered}
$$

where

$$
\gamma(\theta)=\min \left(4 \theta-2, \frac{(8 g-4) \theta-(4 g-3)}{4 g-1}, \frac{24 g \theta-(12 g+1)}{4 g-1}\right) .
$$


Then

$$
\|F P Q\|_{1} \ll x^{1 / 2} \exp \left(-B(\log x)^{1 / 4}\right) .
$$

The implied constant depends on $\eta$ and $g$.

Lemma 5. Let $F\left(s, \lambda^{m}\right), P\left(s, \lambda^{m}\right), Q\left(s, \lambda^{m}\right), R\left(s, \lambda^{m}\right)$ be Dirichlet polynomials with $F P Q R=x$. Suppose that $F, P, Q, R$ satisfy (3.2), and that $F, Q, R$ satisfy (4.1). Also, suppose that $\left(M^{2}+T^{2}\right) \leq x^{1-\theta-\eta}$, where $1 / 2+\eta<\theta \leq 7 / 12$. Then

$$
\|F P Q R\|_{1} \ll x^{1 / 2} \exp \left(-B(\log x)^{1 / 4}\right),
$$

provided that any of the following sets of conditions holds:

(i) $P \gg x^{1-\theta}, Q \gg x^{(1-\theta) / 2}, R \gg x^{(1-\theta) / 4}, F \gg x^{2(1-\theta) / 7}$;

(ii) $P \gg x^{1-\theta}, Q \gg x^{(1-\theta) / 2}, R \gg x^{(1-\theta) / 3}, F \gg x^{2(1-\theta) / 11}$;

(iii) $P \gg x^{1-\theta}, Q \gg x^{(1-\theta) / 3}, R^{2} F \gg x^{1-\theta}, F \gg x^{2(1-\theta) / 5}$;

(iv) $P \gg x^{1-\theta}, Q, R \ll x^{(1-\theta) / 3}, Q R \gg x^{4(1-\theta) / 7}, F Q R \gg x^{14(1-\theta) / 13}$.

Our next result is about polynomials $F\left(s, \lambda^{m}\right)$ in which $c(\mathfrak{a})=1$ for all $\mathfrak{a}$.

Lemma 6. Let

$$
F\left(s, \lambda^{m}\right)=\sum_{\substack{\mathfrak{a} \in \mathcal{C} \\ N \mathfrak{a} \asymp F}} \lambda^{m}(\mathfrak{a})(N \mathfrak{a})^{-s} .
$$

Let $M \leq x, T \leq x, F \leq x$, and suppose that $\mathcal{S}$ contains no pairs $(t, m)$ with $m=0$. Then

$$
\|F\|_{4}^{4} \ll V^{2}(\log x)^{B}+|\mathcal{S}| F^{2} V^{-4}(\log x)^{B},
$$

where $V=M+T$.

Proof. By [17, Theorem 3.19] with $c=\frac{1}{2}+(\log x)^{-1}$,

$$
\begin{array}{r}
F\left(\frac{1}{2}+i t, \lambda^{m}\right)=\frac{1}{2 \pi i} \int_{c-i V}^{c+i V} L\left(w+\frac{1}{2}+i t, \lambda^{m}, \mathcal{C}\right) \frac{F_{2}^{w}-F_{1}^{w}}{w} d w \\
+O\left(F^{1 / 2} V^{-1}(\log x)^{B}\right) ;
\end{array}
$$

here $\left[F_{1}, F_{2}\right]$ is the range of summation in (4.8) and $L\left(s, \lambda^{m}, \mathcal{C}\right)$ is the Hecke $L$ function

$$
L\left(s, \lambda^{m}, \mathcal{C}\right)=\sum_{\mathfrak{a} \in \mathcal{C}} \lambda^{m}(\mathfrak{a})(N \mathfrak{a})^{-s} .
$$

We now move the contour to $[-i V, i V]$. We can prove similarly to [16, Theorem 5$]$ that if $0 \leq \eta \leq \frac{1}{2}$, then

$$
L\left(\sigma+i t, \lambda^{m}, \mathcal{C}\right) \ll\left(t^{2}+m^{2}\right)^{(1-\sigma+\eta) / 2} \zeta^{2}(1+\eta)
$$

whenever $-\eta \leq \sigma \leq 1+\eta$. By this estimate with $\eta=(\log x)^{-1}$, the contribution from the horizontal segments is

$$
\ll \max _{0 \leq \sigma \leq c}\left(\left(m^{2}+V^{2}\right)^{(1-2 \sigma) / 4} F^{\sigma} V^{-1} \log ^{2} x\right) \ll\left(V^{-1 / 2}+F^{1 / 2} V^{-1}\right) \log ^{2} x .
$$

Since the integrand is an entire function, we obtain

$$
F\left(\frac{1}{2}+i t, \lambda^{m}\right)=J\left(t, \lambda^{m}\right)+O\left(\left(F^{1 / 2} V^{-1}+V^{-1 / 2}\right)(\log x)^{B}\right),
$$


where

$$
J\left(t, \lambda^{m}\right)=\frac{1}{2 \pi i} \int_{-i V}^{i V} L\left(w+\frac{1}{2}+i t, \lambda^{m}, \mathcal{C}\right) \frac{F_{2}^{w}-F_{1}^{w}}{w} d w .
$$

To complete the proof, we raise (4.10) to the fourth power, sum over the elements of $\mathcal{S}$, and estimate $\sum_{\mathcal{S}}\left|J\left(t, \lambda^{m}\right)\right|^{4}$, using the approach from [2] Lemma 9] with [4] Theorem 6.3] in place of [2, Lemma 7]. (Also, we have used that $|\mathcal{S}| \ll V^{2}$ to dispense with one of the arising terms.)

Lemma 7. Let $F\left(s, \lambda^{m}\right)$ be defined by 4.8). If $m \neq 0$ and $F \geq\left(m^{2}+t^{2}\right)$, then $\left|F\left(\frac{1}{2}+i t, \lambda^{m}\right)\right| \ll(\log x)^{B}$.

Proof. Let $\left[F_{1}, F_{2}\right]$ be the range for $N \mathfrak{a}$ in (4.8), and let $\psi$ be a $C^{\infty}$-function with the properties:

- $\psi(t)=1$ if $F_{1} \leq t \leq F_{2}$,

- $\psi(t)=0$ if $t \notin\left(F_{1}-\Delta, F_{2}+\Delta\right)$,

- $0 \leq \psi(t) \leq 1$ if $F_{1}-\Delta \leq t \leq F_{2}+\Delta$,

- $\psi^{(j)}(t) \ll j^{2 j} \Delta^{-j}(j=1,2, \ldots)$.

Then, by Mellin's inversion formula,

$$
F\left(\frac{1}{2}+i t, \lambda^{m}\right)=\frac{1}{2 \pi i} \int_{2-i \infty}^{2+i \infty} L\left(w+\frac{1}{2}+i t, \lambda^{m}, \mathcal{C}\right) \widehat{\psi}(w) d w+O\left(\Delta F^{-1 / 2}\right)
$$

where $\widehat{\psi}(w)$ denotes the Mellin transform of $\psi$.

We define the function $\Upsilon\left(s, \lambda^{m}, \mathcal{C}\right)$ via the functional equation

$$
L\left(s, \lambda^{m}, \mathcal{C}\right)=\Upsilon\left(s, \lambda^{m}, \mathcal{C}\right) L\left(1-s, \bar{\lambda}^{m}, \mathcal{C}^{-1}\right) ;
$$

it satisfies the inequality

$$
\Upsilon\left(-\frac{1}{2}+i t, \lambda^{m}, \mathcal{C}\right) \ll\left(m^{2}+t^{2}+1\right) .
$$

Moving the line of integration in (4.11) to $\operatorname{Re} w=-1$ and then using the functional equation (4.12) and (4.13), we deduce that

$$
F\left(\frac{1}{2}+i t, \lambda^{m}\right) \ll \int_{-\infty}^{\infty}\left(m^{2}+t^{2}+v^{2}\right)|\widehat{\psi}(-1+i v)| d v+\Delta F^{-1 / 2} .
$$

Observe that

$$
\widehat{\psi}(\sigma+i t) \ll \Delta F^{\sigma-1}\left(\frac{r^{2} F}{|t| \Delta}\right)^{r} \quad(r=1,2, \ldots) .
$$

We now choose $\Delta=F^{1 / 2}$ and $V=\Delta \log ^{3} x$. Using (4.15) with $r=1$ when $|v| \leq V$ and with $r=[\log x]$ when $|v|>V$, we see that the lemma follows from (4.14).

Lemma 8. Let $P\left(s, \lambda^{m}\right)$ and $Q\left(s, \lambda^{m}\right)$ be Dirichlet polynomials satisfying (3.2), and let $F\left(s, \lambda^{m}\right)$ be defined by (4.8). Suppose that $P Q F=x,\left(M^{2}+T^{2}\right) \leq x^{1-\theta-6 \eta}$, where $1 / 2+\eta<\theta \leq 7 / 12$, and that

$$
\max \left(P, x^{1-\theta}\right) \max \left(Q, x^{(1-\theta) / 2}\right) \leq x^{(1+\theta) / 2+2 \eta} .
$$

Then (4.6) holds. 
Proof. If $F>M^{2}+T^{2}$, using Lemmas 3 and 7 , we have

$$
\begin{aligned}
\|F P Q\|_{1} & \leq\|F\|_{\infty}\|P\|_{2}\|Q\|_{2} \\
& \ll\left(M^{2}+T^{2}+P\right)^{\frac{1}{2}}\left(M^{2}+T^{2}+Q\right)^{\frac{1}{2}}(\log x)^{B} \\
& \ll\left(x^{1-\theta-6 \eta}+x^{(1-\theta-6 \eta) / 2} \max (P, Q)^{\frac{1}{2}}+(x / F)^{\frac{1}{2}}\right)(\log x)^{B} \\
& \ll x^{1 / 2-\eta} .
\end{aligned}
$$

Otherwise, Lemma [6] yields

$$
\|F\|_{4}^{4} \ll\left(M^{2}+T^{2}\right)(\log x)^{B},
$$

whence

$$
\begin{aligned}
\|F P Q\|_{1} & \leq\|P\|_{2}\|Q\|_{4}\|F\|_{4}=\|P\|_{2}\left\|Q^{2}\right\|_{2}^{1 / 2}\|F\|_{4} \\
& \ll\left(M^{2}+T^{2}+P\right)^{\frac{1}{2}}\left(M^{2}+T^{2}+Q^{2}\right)^{\frac{1}{4}}\left(M^{2}+T^{2}\right)^{\frac{1}{4}}(\log x)^{B} \\
& \ll\left(\max \left(P, x^{1-\theta}\right)\right)^{\frac{1}{2}}\left(\max \left(Q, x^{(1-\theta) / 2}\right)\right)^{\frac{1}{2}} x^{(1-\theta-6 \eta) / 4}(\log x)^{B} \\
& \ll x^{1 / 2-\eta / 3} .
\end{aligned}
$$

We conclude this section with two lemmas that provide bounds of the form (4.1).

Lemma 9. Let $z \geq \exp \left(\log ^{7 / 10} x\right)$ and suppose that for all $(t, m) \in \mathcal{S},|t| \ll x^{B}$ and $0<|m| \ll x^{B}$. Then, the Dirichlet polynomial

$$
F\left(s, \lambda^{m}\right)=\sum_{\substack{\mathfrak{a} \in \mathcal{C}, N \mathfrak{a} \asymp F \\(\mathfrak{a}, \mathfrak{P}(z))=1}} \lambda^{m}(\mathfrak{a})(N \mathfrak{a})^{-s}
$$

satisfies (4.1).

Proof. The lemma can be proved similarly to [2, Lemma 5] —one simply has to replace the results on Dirichlet $L$-functions $L(s, \chi)$ used in that proof by the corresponding results on Hecke $L$-functions $L\left(s, \lambda^{m}, \mathcal{C}\right)$. In particular, we use Coleman's zero-free region [5, Theorem 1].

Lemma 10. Let $x^{\varepsilon} \leq F \leq x, 0<|m| \leq x$, and $|t| \leq x$. Then

$$
\sum_{\substack{\mathfrak{a} \in \mathcal{C} \\ N \mathfrak{a} \simeq F}} \lambda^{m}(\mathfrak{a})(N \mathfrak{a})^{-1 / 2-i t} \ll F^{1 / 2-\eta},
$$

where $\eta=\eta(\varepsilon)>0$.

Proof. If $F>\left(m^{2}+t^{2}\right)$, the result follows from Lemma 7 and if $F \leq\left(m^{2}+t^{2}\right)$, we can apply [5, Theorem 6].

4.2. Estimates for polynomials $F(s)$. The estimates from the previous section provide us with the information needed to satisfy hypothesis (3.7) of Lemmas 1 and 2. The results of the present section, on the other hand, will provide information of type (3.8).

Let $2<T \leq x / 4$ and let $\mathcal{S}$ be a set of real numbers $t,|t| \leq T$. Similarly to Section 4.1, we say that $\mathcal{S}$ is well-spaced if $\left|t-t^{\prime}\right| \geq 1$ whenever $t, t^{\prime} \in \mathcal{S}$, and we 
define the norm

$$
\|F\|_{p}= \begin{cases}\left(\sum_{t \in \mathcal{S}}\left|F\left(\frac{1}{2}+i t\right)\right|^{p}\right)^{1 / p}, & 1 \leq p<\infty, \\ \sup _{t \in \mathcal{S}}\left|F\left(\frac{1}{2}+i t\right)\right|, & p=\infty .\end{cases}
$$

Our first result in this section is an analogue of Lemma 3. It is, in fact, a corollary of [2, Lemma 1].

Lemma 11. Let $\mathcal{S}$ be well-spaced, and let $F(s)$ be defined by (3.1) with $m=0$. Also, for an integral ideal $\mathfrak{a}$, let $d(\mathfrak{a})$ denote the number of distinct ideals with norm equal to $N \mathfrak{a}$. Then

$$
\|F\|_{2}^{2} \ll(T+F) G \log x
$$

where

$$
G=G(F)=\sum_{N \mathfrak{a} \asymp F}|c(\mathfrak{a})|^{2} d(\mathfrak{a})(N \mathfrak{a})^{-1} .
$$

Furthermore, if

$$
\left|F\left(\frac{1}{2}+i t\right)\right| \geq V
$$

for all $t \in \mathcal{S}$, then the cardinality of $\mathcal{S}$ is bounded by

$$
|\mathcal{S}| \ll\left(\frac{G F}{V^{2}}+\frac{G^{3} F T}{V^{6}}\right) \log ^{2} x .
$$

Remark. Using Lemma 11 in place of Lemma 3 we can prove versions of Lemmas 4 and 5 in which the Dirichlet polynomials of the form $F\left(s, \lambda^{m}\right)$ have been replaced by polynomials of the form $F(s),\left(M^{2}+T^{2}\right)$ has been replaced by $T$, and the norm from Section 4.1 has been replaced by the norm defined at the beginning of the present section. Thus, each time we refer to Lemmas 4 and 5 to satisfy hypothesis (3.7) of Lemma 1, hypothesis (3.8) will be automatically satisfied as well.

Next, we state variants of Lemmas 6 and 7 . The proofs are analogous, but now the $L$-function $L\left(s, \lambda^{m}, \mathcal{C}\right)$ is replaced by $\zeta_{K}(s, \mathcal{C})$, the zeta-function of the ideal class $\mathcal{C}$, which has a simple pole at $s=1$; hence, the extra terms in the upper bounds.

Lemma 12. Let

$$
F(s)=\sum_{\substack{\mathfrak{a} \in \mathcal{C} \\ N \mathfrak{a} \frown F}}(N \mathfrak{a})^{-s} .
$$

Let $T \leq x$ and $F \leq x$. Then

$$
\|F\|_{4}^{4} \ll T^{2}(\log x)^{B}+|\mathcal{S}| F^{2} T^{-4}(\log x)^{B}+F^{2} \sum_{t \in \mathcal{S}} \frac{1}{1+|t|^{4}} .
$$

Lemma 13. Let $F(s)$ be defined by (4.20). If $F \geq t^{2}$, then

$$
\left|F\left(\frac{1}{2}+i t, \lambda^{m}\right)\right| \ll \frac{F^{1 / 2}}{1+|t|}+(\log x)^{B} .
$$

Lemma 14. Let $P(s), Q(s)$ be Dirichlet polynomials satisfying (3.2), and let $F(s)$ be given by (4.20). Suppose that $P Q F=x$, that

$$
\exp \left(\log ^{1 / 3} x\right) \leq F \leq x \exp \left(-\log ^{1 / 3} x\right),
$$


that $\mathcal{S} \subset[T / 2, T]$, and that

$$
\exp \left(\log ^{1 / 3} x\right) \leq T \leq x^{1-\theta}
$$

where $1 / 2+\eta<\theta \leq 7 / 12$. Then (4.6) holds, provided that

$$
\max (P, T) \max \left(Q, T^{1 / 2}\right) \leq x^{1-\eta} T^{-1} .
$$

Proof. The proof is similar to the proof of Lemma 8 with Lemmas 11, 12 and 13 in place of Lemmas 3, 6 and 7

The final two lemmas of the section are versions of Lemmas 9 and 10 Again, the changes are due to the pole of $\zeta_{K}(s, \mathcal{C})$ at $s=1$.

Lemma 15. Let $z \geq \exp \left(\log ^{7 / 10} x\right)$ and suppose that for all $t \in \mathcal{S}$, we have $\exp \left(\log ^{1 / 3} x\right) \ll|t| \ll x^{B}$. Then the Dirichlet polynomial

$$
F(s)=\sum_{\substack{\mathfrak{a} \in \mathcal{C}, N \mathfrak{a} \asymp F \\(\mathfrak{a}, \mathfrak{P}(z))=1}}(N \mathfrak{a})^{-s}
$$

satisfies (4.1).

Lemma 16. Let $x^{\varepsilon} \leq F \leq x,|t| \leq x$. Then

$$
\sum_{\substack{\mathfrak{a} \in \mathcal{C} \\ N \mathfrak{a} \smile F}}(N \mathfrak{a})^{-1 / 2-i t} \ll F^{1 / 2-\eta}+\frac{F^{1 / 2}}{1+|t|},
$$

where $\eta=\eta(\varepsilon)>0$.

\section{Asymptotic Formulae}

In this section we prove asymptotic formulae of the form (2.4) for various choices of the coefficients $c(\mathfrak{a})$. We will frequently have to consider sums of the form

$$
\sum_{A<N \mathfrak{p}<B} c(\mathfrak{p})
$$

and then introduce further prime ideal variables via Buchstab's identity. When using only rational primes, this leads naturally to sums with conditions expressed by $A<p_{2}<p_{1}<B$. In our present context, we use the ordering of prime ideals employed by Coleman [6], namely $\mathfrak{p}_{2}<\mathfrak{p}_{1}$ if $N \mathfrak{p}_{2}<N \mathfrak{p}_{1}$, or if $N \mathfrak{p}_{2}=N \mathfrak{p}_{1}$ and $\arg \left(\hat{\pi}_{2}\right)<\arg \left(\hat{\pi}_{1}\right)$ where $\hat{\pi}_{j}$ is the ideal number of $\mathfrak{p}_{j}$. With multiple sums it will become very inconvenient to have conditions of the form:

$$
A<N \mathfrak{p}_{1}, N \mathfrak{p}_{2}<B, \mathfrak{p}_{2}<\mathfrak{p}_{1} .
$$

We therefore write, for real $A, B, A<\mathfrak{p}_{1}<B$ to mean $A<N \mathfrak{p}_{1}<B$. Thus (5.1) becomes simply $A<\mathfrak{p}_{1}<\mathfrak{p}_{2}<B$ - exactly the form used for rational primes. We will continue to use $N \mathfrak{p}$ for less complicated expressions, and in places where its use is unavoidable (for example, later we will need to write $\left.\left(x / N\left(\mathfrak{p}_{1} \mathfrak{p}_{2} \mathfrak{p}_{3}\right)\right)^{\frac{1}{2}}\right)$. Furthermore, we will use $\mathfrak{P}\left(\mathfrak{p}_{1}\right)$ to mean

$$
\prod_{\mathfrak{p}_{2}<\mathfrak{p}_{1}} \mathfrak{p}_{2},
$$

and we can then define $\rho(\mathfrak{a}, \mathfrak{p}), S(\mathcal{A}, \mathfrak{p})$ and $S(\mathcal{B}, \mathfrak{p})$ in an analogous fashion to $\rho(\mathfrak{a}, z), S(\mathcal{A}, z)$ and $S(\mathcal{B}, z)$.

We also write $w=\exp \left(\log ^{7 / 10} x\right)$ and $y_{1}=x \exp \left(-3 \log ^{1 / 3} x\right)$. 
Lemma 17. Let $1 / 2+\eta<\theta \leq 7 / 12$, and define

$$
c(\mathfrak{a})=\sum_{\substack{\mathfrak{m} \mathfrak{n} l=\mathfrak{a} \\ N \mathfrak{m} \rightleftharpoons P, N \mathfrak{n} \asymp Q}} a(\mathfrak{m}) b(\mathfrak{n}) \rho(\mathfrak{l}, w) .
$$

Suppose that $a(\mathfrak{m}), b(\mathfrak{n})$ satisfy (3.2) and that $P$ and $Q$ satisfy the condition

$$
\max \left(P, x^{1-\theta}\right) \max \left(Q, x^{(1-\theta) / 2}\right) \leq x^{(1+\theta) / 2} .
$$

Then (3.12) holds.

Proof. We have

$$
c(\mathfrak{a})=\sum_{\mathfrak{m}, \mathfrak{n}} \sum_{\substack{\mathfrak{m} \mathfrak{n} l=\mathfrak{a} \\ \mathfrak{b}|\mathfrak{h}, \mathfrak{b}| \mathfrak{P}(w)}} a(\mathfrak{m}) b(\mathfrak{n}) \mu(\mathfrak{b}),
$$

where for brevity we have suppressed the size conditions on $\mathfrak{m}$ and $\mathfrak{n}$. We set $z=x^{\eta}$ and apply the sieve identity

$$
\sum_{\mathfrak{b}|\mathfrak{l}, \mathfrak{b}| \mathfrak{P}(w)} \mu(\mathfrak{b})=\sum_{\substack{\mathfrak{b}|\mathfrak{l}, \mathfrak{b}| \mathfrak{P}(w) \\ N \mathfrak{b}<z}} \mu(\mathfrak{b})+O\left(\sum_{\substack{\mathfrak{b}|\mathfrak{l}, \mathfrak{b}| \mathfrak{P}(w) \\ z \leq N \mathfrak{b}<w z}} 1\right),
$$

which can be proved similarly to [11, Lemma 15]. We obtain that

$$
\sum_{\mathfrak{a}} c(\mathfrak{a}) \Psi(\mathfrak{a})=\sum_{\mathfrak{a}} c_{1}(\mathfrak{a}) \Psi(\mathfrak{a})+O\left(\sum_{\mathfrak{a}} c_{2}(\mathfrak{a}) \Psi(\mathfrak{a})\right),
$$

where

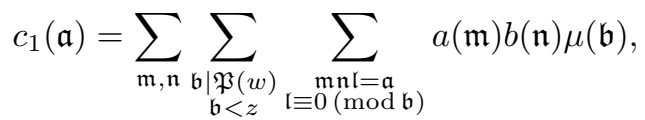

$$
\begin{aligned}
& c_{2}(\mathfrak{a})=\sum_{\mathfrak{m}, \mathfrak{n}} \sum_{\substack{\mathfrak{b} \mid \mathfrak{P}(w) \\
z<\mathfrak{b}<w z}} \sum_{\substack{\mathfrak{m} \mathfrak{n} l=\mathfrak{a} \\
\mathfrak{l}(\bmod \mathfrak{b})}}|a(\mathfrak{m}) b(\mathfrak{n})| .
\end{aligned}
$$

Hence, it suffices to show that

$$
\begin{aligned}
\sum_{\mathfrak{a}} c_{1}(\mathfrak{a}) \Psi(\mathfrak{a}) & =\frac{\phi y}{2 \pi y_{1}} \sum_{\mathfrak{a} \in \mathcal{B}} c_{1}(\mathfrak{a})+O\left(\phi y \exp \left(-(\log x)^{1 / 4}\right)\right), \\
\sum_{\mathfrak{a}} c_{2}(\mathfrak{a}) \Psi(\mathfrak{a}) & =\frac{\phi y}{2 \pi y_{1}} \sum_{\mathfrak{a} \in \mathcal{B}} c_{2}(\mathfrak{a})+O\left(\phi y \exp \left(-(\log x)^{1 / 4}\right)\right), \\
\sum_{\mathfrak{a} \in \mathcal{B}} c_{2}(\mathfrak{a}) & =O\left(y_{1} \exp \left(-(\log x)^{1 / 4}\right)\right) .
\end{aligned}
$$

To prove (5.2), we consider the Dirichlet polynomial

$$
P_{1}\left(s, \lambda^{m}\right) Q\left(s, \lambda^{m}\right) F_{1}\left(s, \lambda^{m}\right),
$$

where

$$
\begin{gathered}
P_{1}\left(s, \lambda^{m}\right)=\sum_{\mathfrak{m}} \sum_{\substack{\mathfrak{b} \mid \mathfrak{P}(w) \\
N \mathfrak{b} \sim D}} a(\mathfrak{m}) \mu(\mathfrak{b}) \lambda^{m}(\mathfrak{m} \mathfrak{b})(N(\mathfrak{m} \mathfrak{b}))^{-s}, \\
Q\left(s, \lambda^{m}\right)=\sum_{\mathfrak{n}} b(\mathfrak{n}) \lambda^{m}(\mathfrak{n})(N \mathfrak{n})^{-s}, \quad F_{1}\left(s, \lambda^{m}\right)=\sum_{\mathfrak{l} \asymp F D^{-1}} \lambda^{m}(\mathfrak{l})(N \mathfrak{l})^{-s},
\end{gathered}
$$


with $D$ of the form $D=z 2^{-j}, 0 \leq j \ll \log x$, and $\mathfrak{b}, \mathfrak{l}, \mathfrak{m}, \mathfrak{n}$ belonging to fixed ideal classes. Split the sum on the left-hand side of (5.2) into subsums with $N \mathfrak{b} \sim D$ and with $\mathfrak{b}, \mathfrak{l}, \mathfrak{m}, \mathfrak{n}$ in fixed ideal classes with $\mathfrak{b} \mathfrak{l m n} \in \mathcal{C}$. If $P Q D \geq x^{\eta}$, we can apply Lemma 1 to the corresponding subsum. Mean-value estimates of the forms (3.7) and (3.8) are provided by Lemmas 8 and 14, respectively. If $P Q D \leq x^{\eta}$, we use Lemma 2 and Lemma 8 to satisfy (3.7). Summing the resulting asymptotic formulae over $D$ and the appropriate ideal classes, we complete the proof of $(5.2)$. The proof of (5.3) is similar.

Finally, consider (5.4). Using (3.17), we get

$$
\sum_{\mathfrak{a} \in \mathcal{B}} c_{2}(\mathfrak{a}) \ll y_{1} \sum_{\mathfrak{m}, \mathfrak{n}} \frac{|a(\mathfrak{m}) b(\mathfrak{n})|}{N(\mathfrak{m} \mathfrak{n})} \sum_{\substack{\mathfrak{b} \mid \mathfrak{P}(w) \\ N \mathfrak{b}>z}} \frac{1}{N \mathfrak{b}} .
$$

Since one can show similarly to [3. Lemma 7] that

$$
\sum_{\substack{\mathfrak{b} \mid \mathfrak{P}(w) \\ N \mathfrak{b} \geq z}} \frac{1}{N \mathfrak{b}} \ll \exp \left(-0.1 \eta(\log x)^{3 / 10} \log \log x\right),
$$

(5.4) follows from (5.5) and the properties of the coefficients $a(\mathfrak{m}), b(\mathfrak{n})$.

Lemma 17 is a "fundamental lemma". The next step is to use this lemma to obtain a similar result in which $w$ is replaced by a larger quantity. We will accomplish this by means of a combinatorial identity that is a variation of Buchstab's identity:

$$
\rho\left(\mathfrak{l}, z_{1}\right)=\rho\left(\mathfrak{l}, z_{2}\right)-\sum_{\substack{\mathfrak{p} \mathfrak{b}=\mathfrak{l} \\ z_{2} \leq N \mathfrak{p}<z_{1}}} \rho(\mathfrak{b}, \mathfrak{p}) \quad\left(2 \leq z_{2}<z_{1}\right) .
$$

Lemma 18. Let $1 / 2+\eta \leq \theta \leq 7 / 12$, and define

$$
c(\mathfrak{a})=\sum_{\substack{\mathfrak{m} \mathfrak{n} l=\mathfrak{a} \\ N \mathfrak{m} \sim P, N \mathfrak{n} \sim Q}} a(\mathfrak{m}) b(\mathfrak{n}) \rho(\mathfrak{l}, z),
$$

where $a(\mathfrak{m}), b(\mathfrak{n})$ satisfy $(3.2)$. Let $2 P=x^{\alpha}, Q=x^{\beta}$, and let $h$ be the positive integer satisfying

$$
1 / 2-h(2 \theta-1) \leq \alpha<1 / 2-(h-1)(2 \theta-1) .
$$

Define

$$
\alpha^{*}=\max \left(\frac{2 h(1-\theta)-\alpha}{2 h-1}, \frac{2(h-1) \theta+\alpha}{2 h-1}\right),
$$

and suppose that

$$
0 \leq \alpha \leq 1 / 2, \quad 0 \leq \beta \leq \frac{1+\theta}{2}-\alpha^{*}=\beta^{*}(\alpha) \quad \text { say }
$$

Then (3.12) holds whenever $z \leq x^{\nu(\alpha)}$, where

$$
\nu(\alpha)=\min \left(\frac{2}{2 h-1}(\theta-\alpha), \gamma(\theta)\right)
$$

and $\gamma(\theta)$ is the function appearing on the right side of 4.5.

Notice that $\nu(\alpha) \geq 2 \theta-1$ and that the upper bound for $\beta$ is $\geq \theta / 2$. We also remark that for the proof of Theorem 2 we shall take $g=4$ in the definition of $\gamma(\theta)$. This yields $\gamma(\theta)=4 \theta-2$ for $19 / 36 \leq \theta \leq 17 / 32$, in particular for $\theta=0.53$. 
Proof. From (5.6),

$$
c(\mathfrak{a})=c_{0}^{\prime}(\mathfrak{a})-c^{*}(\mathfrak{a}),
$$

where

$$
\begin{aligned}
c_{0}^{\prime}(\mathfrak{a}) & =\sum_{\mathfrak{m} \mathfrak{n} \mathfrak{l}=\mathfrak{a}} a(\mathfrak{m}) b(\mathfrak{n}) \rho(\mathfrak{l}, w), \\
c^{*}(\mathfrak{a}) & =\sum_{\substack{\mathfrak{m} \mathfrak{n} \mathfrak{p}_{1} \mathfrak{b}_{1}=\mathfrak{a} \\
w \leq \mathfrak{p}_{1}<z}} a(\mathfrak{m}) b(\mathfrak{n}) \rho\left(\mathfrak{b}_{1}, \mathfrak{p}_{1}\right) .
\end{aligned}
$$

We split $c^{*}(\mathfrak{a})$ into two subsums:

$$
c^{*}(\mathfrak{a})=c_{0}^{\prime \prime}(\mathfrak{a})+c_{1}(\mathfrak{a}),
$$

where $c_{0}^{\prime \prime}(\mathfrak{a})$ and $c_{1}(\mathfrak{a})$ are subject to $N \mathfrak{m}\left(N \mathfrak{p}_{1}\right)^{1 / 2}>x^{1-\theta}$ and $N \mathfrak{m}\left(N \mathfrak{p}_{1}\right)^{1 / 2} \leq x^{1-\theta}$, correspondingly. We now use (5.6) to decompose $c_{1}(\mathfrak{a})$. In general, if

$$
c_{j}(\mathfrak{a})=\sum_{\substack{\mathfrak{m} \mathfrak{p}_{1} \cdots \mathfrak{p}_{\mathfrak{j}} \mathfrak{b}_{j}=\mathfrak{a} \\ w \leq \mathfrak{p}_{j}<\cdots<\mathfrak{p}_{1}<z}} a(\mathfrak{m}) b(\mathfrak{n}) \rho\left(\mathfrak{b}_{j}, \mathfrak{p}_{j}\right)
$$

is subject to

$$
N\left(\mathfrak{m} \mathfrak{p}_{1} \cdots \mathfrak{p}_{j-1}\right)\left(N \mathfrak{p}_{j}\right)^{1 / 2} \leq x^{1-\theta}
$$

then (5.6) gives

$$
c_{j}(\mathfrak{a})=c_{j}^{\prime}(\mathfrak{a})-c_{j}^{\prime \prime}(\mathfrak{a})-c_{j+1}(\mathfrak{a}),
$$

where $c_{j}^{\prime}(\mathfrak{a})$ is obtained from $c_{j}(\mathfrak{a})$ by replacing $\rho\left(\mathfrak{b}_{j}, \mathfrak{p}_{j}\right)$ by $\rho\left(\mathfrak{b}_{j}, w\right)$, and $c_{j}^{\prime \prime}(\mathfrak{a})$ is $c_{j+1}(\mathfrak{a})$ with condition (5.7) replaced by

$$
N\left(\mathfrak{m} \mathfrak{p}_{1} \cdots \mathfrak{p}_{j-1}\right)\left(N \mathfrak{p}_{j}\right)^{1 / 2} \leq x^{1-\theta}<N\left(\mathfrak{m} \mathfrak{p}_{1} \cdots \mathfrak{p}_{j}\right)\left(N \mathfrak{p}_{j+1}\right)^{1 / 2} .
$$

Clearly, this process has to stop after $\ll \log x$ steps, that is, for some $j \ll \log x$, we will be left with an empty $c_{j+1}(\mathfrak{a})$.

We now show that we can find asymptotic formulae for all sums $c_{j}^{\prime}(\mathfrak{a})$ and $c_{j}^{\prime \prime}(\mathfrak{a})$. We consider $c_{j}^{\prime}(\mathfrak{a})$ first. Combining (5.7) and (5.9) by means of a simple argument (see [3, Lemma 10] for details), we have

$$
N\left(\mathfrak{m} \mathfrak{p}_{1} \cdots \mathfrak{p}_{j}\right) \leq x^{\alpha^{*}} .
$$

Since $\alpha^{*} \geq 1-\theta$, we can show that (3.12) holds for $c_{j}^{\prime}(\mathfrak{a})$ by an appeal to Lemma 17 with $a(\mathfrak{m})$ replaced by

$$
\sum_{\substack{\mathfrak{m}, \mathfrak{p}_{1}, \ldots, \mathfrak{p}_{j}:(\mathbb{5}, \mathfrak{g}) \\ w \leq \mathfrak{p}_{j}<\cdots<\mathfrak{p}_{1}<z}} a(\mathfrak{m}) .
$$

To show that $c_{j}^{\prime \prime}(\mathfrak{a})$ satisfies (3.12), we combine Lemma 1, Lemma 4, and the Remark after Lemma 11. In the application of Lemma 4, $P\left(s, \lambda^{m}\right)$ is obtained by grouping the variables $\mathfrak{m}, \mathfrak{p}_{1}, \ldots, \mathfrak{p}_{j}$, while $F\left(s, \lambda^{m}\right)$ corresponds to the summation over $\mathfrak{p}_{j+1}$, and $Q\left(s, \lambda^{m}\right)$ comes from grouping the remaining variables. The restriction to dyadic ranges is not a problem. Inequalities (4.4) and (4.5) follow from the hypotheses of the lemma and an elementary argument (cf. [3, Lemma 11]), and Lemma 9 provides the "local bound" (4.1). Thus, Lemma 4 would be applicable if the summation variables in the polynomials $P\left(s, \lambda^{m}\right), Q\left(s, \lambda^{m}\right)$, and $F\left(s, \lambda^{m}\right)$ were 
independent of each other (which unfortunately they are not). We complete the proof of the lemma by showing how one can use Perron's formula to remove the interdependencies between the variables at the cost of multiplying the bounds for some error terms by a factor of $(\log x)^{B}$.

Suppose that the summation condition $\mathfrak{p}_{j+1}<\mathfrak{p}_{j}$ and the weight $\rho\left(\mathfrak{b}_{j+1}, \mathfrak{p}_{j+1}\right)$ are the only relations between variables that need to be removed (additional summation conditions can be removed by reiterating the same procedure). We have

$$
\rho\left(\mathfrak{b}_{j+1}, \mathfrak{p}_{j+1}\right)=\sum_{\mathfrak{l} \mid\left(\mathfrak{b}_{j+1}, \mathfrak{P}\left(\mathfrak{p}_{j+1}\right)\right)} \mu(\mathfrak{l})=1-\sum_{2 \leq \mathfrak{p}<\mathfrak{p}_{j+1}} \sum_{\mathfrak{l} \mid \mathfrak{P}(\mathfrak{p})} \mu(\mathfrak{l}) ;
$$

so we really need to worry only about inequalities like $\mathfrak{p}_{j+1}<\mathfrak{p}_{j}$. Using Perron's formula [9, eq. (15)], we obtain

$$
c_{j}^{\prime \prime}(\mathfrak{a})=\frac{1}{\pi} \int_{-Y}^{Y} \sum_{\substack{\mathfrak{m}, \mathfrak{n} \mathfrak{p}_{1}, \ldots, \mathfrak{p}_{j}, \mathfrak{b}_{j+1} \\ \mathfrak{m} \mathfrak{n} \mathfrak{p}_{1} \cdots \mathfrak{p}_{j+1} \mathfrak{b}_{j+1}=\mathfrak{a}}} \sum_{\substack{\mathfrak{p}_{j+1} \\ \mathfrak{y}_{j}}}^{*} a(\mathfrak{m}) b(\mathfrak{n}) d\left(\mathfrak{p}_{j}, u\right)\left(N \mathfrak{p}_{j+1}\right)^{i u} \frac{d u}{u}+O\left(x^{-2}\right),
$$

where $Y=x^{B}, d\left(\mathfrak{p}_{j}, u\right) \ll \min (1,|u|)(\log x)^{B}$, and the asterisk means that the condition $\mathfrak{p}_{j+1}<\mathfrak{p}_{j}$ has been removed. Assume that the last sum is free of unwanted dependencies between the variables. The contribution from $|u|<Y^{-1}$ can be estimated trivially as $O\left(x^{-2}\right)$; so it suffices to establish that (3.7) holds for the Dirichlet polynomial corresponding to the coefficients

$$
c_{j}^{*}(\mathfrak{a})=\frac{1}{\pi} \int_{U / 2}^{U} \sum_{\substack{\mathfrak{m}, \mathfrak{n} \\ \mathfrak{m} \mathfrak{\mathfrak { p } _ { 1 }}, \ldots, \mathfrak{p}_{j}, \mathfrak{b}_{j+1} \cdots \mathfrak{p}_{j+1} \mathfrak{b}_{j+1}=\mathfrak{a}}} \sum_{\mathfrak{p}_{j+1}}^{*} a(\mathfrak{m}) b(\mathfrak{n}) d\left(\mathfrak{p}_{j}, u\right)\left(N \mathfrak{p}_{j+1}\right)^{i u} \frac{d u}{u},
$$

where $Y^{-1} \leq|U| \leq Y$. Let $c_{j}^{*}(\mathfrak{a}, u)$ denote the integrand in the definition of $c_{j}^{*}(\mathfrak{a})$. For each fixed $u$, we can use Lemmas 1, 4, and 9 as we intended initially, since the Dirichlet polynomial corresponding to $c_{j}^{*}(\mathfrak{a}, u)$ is of the form $P(s) Q(s) F(s-i u)$ with $P, Q$, and $F$ as desired. (It is crucial for this step that the hypotheses of Lemma 9 do not include a lower bound for the imaginary parts of the elements of $\mathcal{S}$.) By interchanging the order of averaging over $(m, t)$ and $u$, we see that the sequence $c_{j}^{*}(\mathfrak{a})$ satisfies hypothesis (3.7) of Lemma 1.

In order to show that $c_{j}^{*}(\mathfrak{a})$ satisfies 3.8 , we need to work more carefully. After interchanging the integrations over $t$ and $u$, we partition $\left[T_{0}, T_{1}\right]$ into two subsets:

$$
E_{1}(u)=\left\{t:|t-u| \leq \exp \left(B(\log x)^{1 / 4}\right)\right\}, \quad E_{2}(u)=\left[T_{0}, T_{1}\right]-E_{1}(u) .
$$

We can estimate the contribution from $E_{1}(u)$ as before, using Lemma 15 to satisfy (4.1). Let $R(s, u)$ be the Dirichlet polynomial with coefficients $c_{j}^{*}(\mathfrak{a}, u)$. The contribution from $E_{2}(u)$ is

$$
\ll \iint\left|R\left(\frac{1}{2}+i t, u_{0}\right)\right| d t \frac{d u}{u},
$$

where $u_{0} \in[U / 2, U]$ is fixed and the integration is over $t$ and $u$ subject to

$$
U / 4 \leq t, u \leq 2 U, \quad|t-u| \leq \exp \left(B(\log x)^{1 / 4}\right) .
$$

We now observe that if we replace (4.1) by the weaker hypothesis

$$
\|F\|_{\infty} \ll x^{1 / 2}(\log x)^{B}
$$




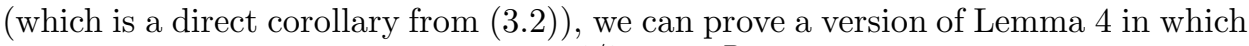
the right side of (4.6) is replaced by $x^{1 / 2}(\log x)^{B}$. Hence, the integral in (5.10) is

$$
\ll T_{0}^{-1} \exp \left(B(\log x)^{1 / 4}\right) x^{1 / 2}(\log x)^{B} \ll x^{1 / 2} \exp \left(-B(\log x)^{1 / 4}\right) .
$$

This completes the proof that $c_{j}^{*}(\mathfrak{a})$ satisfies (3.8)).

\section{The FINAL DECOMPOSITION}

Write

$$
z(\mathfrak{n})=x^{\nu(\alpha)} \text { where } 2^{j} \leq N \mathfrak{n}<2^{j+1}=x^{\alpha} .
$$

Also, put $\nu_{0}=2 \theta-1, z_{0}=x^{\nu_{0}}$. Our initial Buchstab decomposition is as follows:

$$
\begin{aligned}
S\left(\mathcal{A}, x^{1 / 2}\right) & =S\left(\mathcal{A}, z_{0}\right)-\sum_{z_{0} \leq \mathfrak{p}<x^{1 / 2}} S\left(\mathcal{A}_{\mathfrak{p}}, z(\mathfrak{p})\right)+\sum_{z\left(\mathfrak{p}_{1}\right) \leq \mathfrak{p}_{2}<\mathfrak{p}_{1}<x^{1 / 2}} S\left(\mathcal{A}_{\left.\mathfrak{p}_{1} \mathfrak{p}_{2}, \mathfrak{p}_{2}\right)}\right. \\
& =S_{1}-S_{2}+S_{3} \quad \text { say. }
\end{aligned}
$$

By Lemma 18 we can give an asymptotic formula for $S_{1}$ and $S_{2}$. We can also give an asymptotic formula for those parts of $S_{3}$ with $N \mathfrak{p}_{2}<z\left(\mathfrak{p}_{1} \mathfrak{p}_{2}\right)$. To consider the remainder of $S_{3}$, we introduce a device familiar from the method applied to rational primes (see 1, 2, 3, 9). We start by writing $N \mathfrak{p}_{j}=x^{\alpha_{j}}$. Then, using techniques analogous to the rational prime case (with the Prime Ideal Theorem replacing the Prime Number Theorem) we may convert sums over primes to integrals. For example,

$$
\sum_{\substack{z_{0} \leq \mathfrak{p}_{2}<\mathfrak{p}_{1}<x^{1 / 2} \\ \mathfrak{p}_{1} \mathfrak{p}_{2}^{2}<x}} S\left(\mathcal{B}_{\mathfrak{p}_{1} \mathfrak{p}_{2}}, \mathfrak{p}_{2}\right)=S\left(\mathcal{B}, x^{1 / 2}\right)(I+o(1)),
$$

where

$$
I=\int_{\nu_{0}}^{\frac{1}{2}} \int_{\nu_{0}}^{\min \left(\alpha_{1}, \frac{1}{2}\left(1-\alpha_{1}\right)\right)} w\left(\frac{1-\alpha_{1}-\alpha_{2}}{\alpha_{2}}\right) \frac{d \alpha_{2} d \alpha_{1}}{\alpha_{1} \alpha_{2}^{2}}
$$

and $w(t)$ is Buchstab's function, which is defined for $t \geq 1$ as the continuous solution of the differential delay equation

$$
\begin{cases}w(t)=1 / t & \text { if } 1 \leq t \leq 2 \\ (t w(t))^{\prime}=w(t-1) & \text { if } t>2\end{cases}
$$

From this definition it follows that

$$
w(t) \begin{cases}=1 / u & \text { if } 1 \leq u \leq 2 \\ =(1+\log (u-1)) / u & \text { if } 2 \leq u \leq 3 \\ \leq \frac{1}{3}(1+\log 2) & \text { if } u \geq 3\end{cases}
$$

Our method ensures that we will only ever be concerned with upper bounds for integrals; so we can use the above approximation to $w(u)$ where appropriate.

The expressions that will usually arise in the following have the form

$$
\sum_{\mathfrak{m}_{j} \sim x^{\alpha_{j}}} S\left(\mathcal{A}_{\mathfrak{m}_{1} \ldots \mathfrak{m}_{n}}, \mathfrak{m}_{n}\right)
$$

where all the prime factors of each $\mathfrak{m}_{j}$ exceed $z_{0}$, and there may be certain interdependencies between the variables. We write

$$
G_{n}=\left\{\left(\alpha_{1}, \ldots, \alpha_{n}\right) \in \mathbb{R}^{n}: \text { an asymptotic formula can be obtained for (6.1) }\right\} .
$$


In addition, we let

$$
D=\left\{(\alpha, \beta): \nu_{0} \leq \alpha \leq \frac{1}{2}, \nu_{0} \leq \beta \leq \beta^{*}(\alpha)\right\}
$$

The significance of $D$ ( $D$ for "decomposable") is that if we have a sum of the form (6.1) and we can partition $\alpha_{1}, \ldots, \alpha_{n}$ into two sets whose sums are $\alpha, \beta$ with $(\alpha, \beta) \in D$, then we can apply Buchstab's identity followed by Lemma 18. Usually we will need to do this twice; so we want to be able to partition $\alpha_{1}, \ldots, \alpha_{n}, \gamma$ into two sets whose sums are $(\alpha, \beta) \in D$ for all $\gamma \in\left[\nu_{0}, \alpha_{n}\right]$.

We treat $S_{3}$ by considering $\left(\alpha_{1}, \alpha_{2}\right)$ belonging to the following disjoint sets whose union is $\left\{\left(\alpha_{1}, \alpha_{2}\right): \nu_{0} \leq \alpha_{1} \leq \frac{1}{2}, \nu\left(\alpha_{1}\right) \leq \alpha_{2} \leq \alpha_{1}\right\}$ :

$$
\begin{aligned}
& T_{1}=\left\{\left(\alpha_{1}, \alpha_{2}\right): \frac{1}{3} \leq \alpha_{1} \leq \frac{1}{2},\left(1-\alpha_{1}\right) / 2 \leq \alpha_{2} \leq \alpha_{1}\right\} \\
& T_{2}=\left\{\left(\alpha_{1}, \alpha_{2}\right): \theta / 2 \leq \alpha_{1} \leq 1-\theta, \theta / 2 \leq \alpha_{2} \leq \min \left(\alpha_{1},\left(1-\alpha_{1}\right) / 2\right)\right\} ; \\
& A_{1}=\left\{\left(\alpha_{1}, \alpha_{2}\right): \nu_{0} \leq \alpha_{1} \leq \frac{1}{4}, \nu\left(\alpha_{1}\right) \leq \alpha_{2} \leq \alpha_{1}\right\} \\
& A_{2}=\left\{\left(\alpha_{1}, \alpha_{2}\right): \frac{1}{4}<\alpha_{1} \leq \frac{2}{5}, \nu\left(\alpha_{1}\right) \leq \alpha_{2} \leq\left(1-\alpha_{1}\right) / 3\right\} ; \\
& A_{3}=\left\{\left(\alpha_{1}, \alpha_{2}\right): \frac{2}{5}<\alpha_{1} \leq \frac{1}{2}, \nu\left(\alpha_{1}\right) \leq \alpha_{2} \leq \alpha_{1} / 2\right\} ; \\
& B_{1}=\left\{\left(\alpha_{1}, \alpha_{2}\right): \frac{1}{4} \leq \alpha_{1} \leq \frac{2}{5},\left(1-\alpha_{1}\right) / 3<\alpha_{2} \leq \min \left(\theta / 2, \alpha_{1}, 1-2 \alpha_{1}\right)\right\} ; \\
& B_{2}=\left\{\left(\alpha_{1}, \alpha_{2}\right):(2-\theta) / 4 \leq \alpha_{1} \leq \frac{1}{2},\right.
\end{aligned}
$$

Region $T_{1}$ corresponds to products $\mathfrak{p}_{1} \mathfrak{p}_{2}$ with $\mathfrak{p}_{2}<\mathfrak{p}_{1}<x^{1 / 2}$. Since the trivial estimate for the number of such products in $\mathcal{B}$ is $O\left(y_{1}(\log x)^{-2}\right)$, discarding $T_{1}$ will not affect the final bound. Although it is possible to apply Buchstab's identity twice more to a large part of region $T_{2}$ (using a two-dimensional sieve as in 3 ), we lose practically nothing in discarding the whole set. The problem that arises is

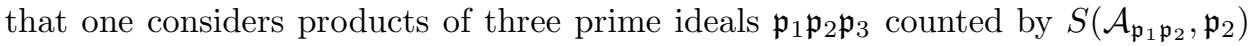
and one has to decompose both $\mathfrak{p}_{1}$ and $\mathfrak{p}_{3}$. A large proportion of the resulting sums is inaccessible to our methods for obtaining asymptotic formulae, and so the ensuing four-dimensional loss exceeds the original two-dimensional loss. We therefore discard a sum of size

$$
\frac{\phi y}{2 \pi} \delta S\left(\mathcal{B}, x^{\frac{1}{2}}\right)(1+o(1))
$$

where

$$
\delta=\int_{\left(\alpha_{1}, \alpha_{2}\right) \in T_{2}} \frac{d \alpha_{2} d \alpha_{1}}{\alpha_{1} \alpha_{2}\left(1-\alpha_{1}-\alpha_{2}\right)}<0.2 \text { at } \theta=0.53 .
$$

The reader should note that both the regions $B_{1}$ and $B_{2}$ correspond to products of three prime ideals and $\left(\alpha_{1}, \alpha_{2}\right) \in B_{1} \Leftrightarrow\left(1-\alpha_{1}-\alpha_{2}, \alpha_{2}\right) \in B_{2}$. It therefore suffices to consider only one of these regions during the calculations.

Now suppose that $R$ is any subset of $A_{j}$ or $B_{k}$. We will show that it is always possible to apply Buchstab's identity twice more to a sum

$$
\sum_{\left(\alpha_{1}, \alpha_{2}\right) \in R} S\left(\mathcal{A}_{\mathfrak{p}_{1} \mathfrak{p}_{2}}, \mathfrak{p}_{2}\right)
$$


Since $\alpha_{2} \leq \theta / 2$ and $\alpha_{1} \leq \frac{1}{2}$ we can decompose (6.3) as

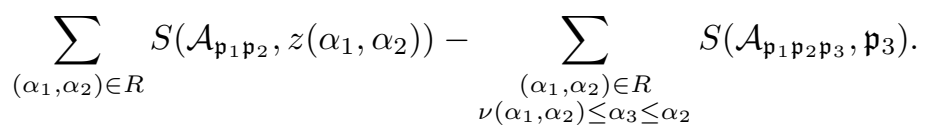

Here

$$
\nu\left(\alpha_{1}, \alpha_{2}\right)=\max \left(\nu\left(\alpha_{1}\right), \nu\left(\alpha_{1}+\alpha_{2}\right)\right), \quad z\left(\alpha_{1}, \alpha_{2}\right)=x^{\nu\left(\alpha_{1}, \alpha_{2}\right)},
$$

with the convention that $\nu(\gamma)=0$ for $\gamma>\frac{1}{2}$. Now Lemma 18 gives an asymptotic formula for the first sum in (6.4). To consider the second sum, we split into two cases. If $\alpha_{1}+\alpha_{3} \leq \frac{1}{2}$ or $\alpha_{2}+\alpha_{3} \leq \beta^{*}\left(\alpha_{1}\right)$ (call this case $F_{1}$ for future reference), then we can apply Buchstab's identity once more in a straightforward fashion. On the other hand, if $\alpha_{1}+\alpha_{3}>\frac{1}{2}$ and $\alpha_{2}+\alpha_{3}>\beta^{*}\left(\alpha_{1}\right)$ (we will call this region $F_{2}$ for future reference), we reverse the rôles of two of the ideals. To be explicit,

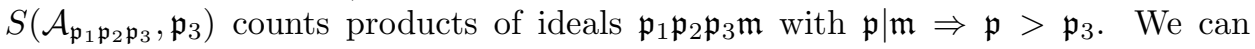
represent this by

$$
S\left(\mathcal{A}_{\mathfrak{p}_{2} \mathfrak{p}_{3} \mathfrak{m}},\left(x / N\left(\mathfrak{p}_{2} \mathfrak{p}_{3} \mathfrak{m}\right)\right)^{1 / 2}\right) .
$$

We now have $N\left(\mathfrak{p}_{2} \mathfrak{m}\right)<x^{1 / 2}$ and $N \mathfrak{p}_{3} \leq x^{\theta / 2}$. We can therefore apply Buchstab's identity and use Lemma 18 . We thus have (with $N \mathfrak{m} \sim x^{1-\alpha_{1}-\alpha_{2}-\alpha_{3}}$ )

$$
\begin{aligned}
& \sum_{\substack{\left(\alpha_{1}, \alpha_{2}\right) \in R \\
\left(\alpha_{1}, \alpha_{2}\right) \leq \alpha_{3} \leq \alpha_{2}}} S\left(\mathcal{A}_{\left.\mathfrak{p}_{1} \mathfrak{p}_{2} \mathfrak{p}_{3}, \mathfrak{p}_{3}\right)}\right.
\end{aligned}
$$

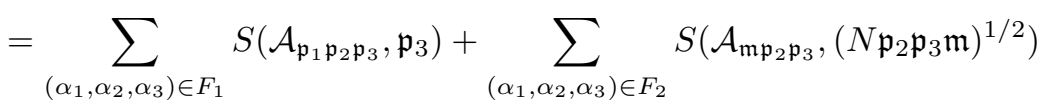

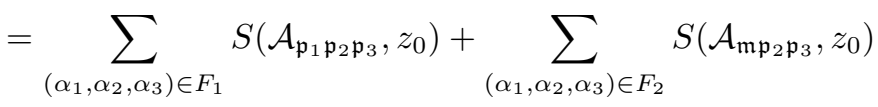

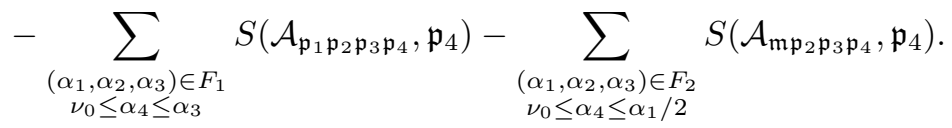

We give asymptotic formulae for the first two sums on the right-hand side of (6.5) by Lemma 18 as indicated above, and possibly, for some part of the final two sums, we have $\left(\alpha_{1}, \ldots, \alpha_{4}\right) \in G_{4}$. Taking into account the possibility that the losses from the final sums in (6.5) may be larger than the losses from the original two-dimensional sum, we are left with a corresponding loss

$$
\int_{R} \min \left(w\left(\frac{1-\alpha_{1}-\alpha_{2}}{\alpha_{2}}\right) \frac{1}{\alpha_{2}}, I_{1}\left(\alpha_{1}, \alpha_{2}\right)+I_{2}\left(\alpha_{1}, \alpha_{2}\right)\right) \frac{d \alpha_{2} d \alpha_{1}}{\alpha_{1} \alpha_{2}},
$$

where

$$
\begin{aligned}
& I_{1}\left(\alpha_{1}, \alpha_{2}\right)=\int_{R_{1}} w\left(\frac{1-\alpha_{1}-\alpha_{2}-\alpha_{3}-\alpha_{4}}{\alpha_{4}}\right) \frac{d \alpha_{4} d \alpha_{3}}{\alpha_{3} \alpha_{4}^{2}} \\
& I_{2}\left(\alpha_{1}, \alpha_{2}\right)=\alpha_{1} \int_{R_{2}} w\left(\frac{\alpha_{1}-\alpha_{4}}{\alpha_{4}}\right) w\left(\frac{1-\alpha_{1}-\alpha_{2}-\alpha_{3}}{\alpha_{3}}\right) \frac{d \alpha_{4} d \alpha_{3}}{\alpha_{3}^{2} \alpha_{4}^{2}},
\end{aligned}
$$

with

$$
\begin{aligned}
& R_{1}=\left\{\left(\alpha_{1}, \ldots, \alpha_{4}\right) \notin G_{4}:\left(\alpha_{1}, \alpha_{2}, \alpha_{3}\right) \in F_{1}, \nu_{0} \leq \alpha_{4} \leq \alpha_{3}\right\} \\
& R_{2}=\left\{\left(\alpha_{1}, \ldots, \alpha_{4}\right) \notin G_{4}:\left(\alpha_{1}, \alpha_{2}, \alpha_{3}\right) \in F_{2}, \nu_{0} \leq \alpha_{4} \leq \alpha_{1} / 2\right\}
\end{aligned}
$$


Now, (6.6) may already be starting to look a little ungainly, but we can repeat the ideas used so far and introduce other possibilities to reduce the size of the integral. Rather than expand (6.6) explicitly (thus sparing the reader a very complicated expression!), we mention the main two devices for reducing the proportion of $S_{3}$ discarded.

1) Further decompositions. So long as $\left(\alpha_{1}, \ldots, \alpha_{4}, \gamma\right) \in D$ for all $\gamma \in\left[\nu_{0}, \alpha_{4}\right]$, we can perform two more decompositions to arrive at a six-dimensional integral. Indeed, we can perform even more Buchstab decompositions for parts of the resulting sums. The contribution from 8 or more decompositions is extremely small even before removing the contribution from $\left(\alpha_{1}, \ldots, \alpha_{8}\right) \in G_{8}$. This can be seen by noting that

$$
\int_{\nu_{0}}^{\lambda} \int_{\nu_{0}}^{\alpha_{1}} \ldots \int_{\nu_{0}}^{\alpha_{7}} \frac{d \alpha_{8} \ldots d \alpha_{1}}{\alpha_{8}^{2} \ldots \alpha_{1}} \leq \frac{\log ^{8}(\lambda / \nu)}{8 ! \nu_{0}}
$$

$\left(\left(8 ! \nu_{0}\right)^{-1} \approx 4.13 \times 10^{-4}\right.$ for $\left.\theta=0.53\right)$.

2) Expanding $S\left(\mathcal{A}_{\mathfrak{p}_{1} \ldots \mathfrak{p}_{n}}, \mathfrak{p}_{n}\right)$. We can make explicit the almost-primes counted by the sifting function $S\left(\mathcal{A}_{\mathfrak{p}_{1} \ldots \mathfrak{p}_{n}}, \mathfrak{p}_{n}\right)$ using Buchstab's identity in reverse:

$$
\begin{aligned}
& S\left(\mathcal{A}_{\mathfrak{p}_{1} \ldots \mathfrak{p}_{n}}, \mathfrak{p}_{n}\right)=S\left(\mathcal{A}_{\mathfrak{p}_{1} \ldots \mathfrak{p}_{n}},\left(x / N \mathfrak{p}_{1} \ldots \mathfrak{p}_{n}\right)^{1 / 2}\right) \\
& +\sum_{\mathfrak{p}_{n}<\mathfrak{p}_{n+1}<\left(x / N \mathfrak{p}_{1} \ldots \mathfrak{p}_{n}\right)^{1 / 2}} S\left(\mathcal{A}_{\mathfrak{p}_{1} \ldots \mathfrak{p}_{n+1}}, \mathfrak{p}_{n+1}\right) \text {. }
\end{aligned}
$$

We would only be applying this technique if we could not give an asymptotic formula for the left-hand side of (6.7); so we cannot give a formula for the first term on the right-hand side. However, we may be able to give a formula for some of the second sum. Clearly we could apply the technique again to this term.

Working in this way leads to the following results for $\theta=0.53$ :

$\begin{array}{cc}\text { Region } & \text { Upper bound for loss } \\ A_{1} & 0.063 \\ A_{2} & 0.183 \\ A_{3} & 0.25 \\ B_{1} & 0.12 \\ B_{2} & 0.12\end{array}$

Combining this with (6.2) gives an overall loss of no more than 0.936. Since the loss is a continuous function of $\theta$, we obtain

$$
\sum_{\mathfrak{p} \in \mathcal{A}} 1 \geq \frac{\phi y}{20 \log x}
$$

for all $\theta \geq 0.53-2 \varepsilon$ and for all $x>x_{0}(\varepsilon)$, which completes the proof.

\section{REFERENCES}

1. R. C. Baker and G. Harman, The difference between consecutive primes, Proc. London Math. Soc. (3) 72 (1996), 261-280. MR 96k:11111

2. R. C. Baker, G. Harman and J. Pintz, The exceptional set for Goldbach's problem in short intervals, in "Sieve Methods, Exponential Sums and their Applications in Number Theory", London Math. Soc. Lecture Notes 237, Cambridge University Press, 1997, pp. 1-54. MR 99g:11121

3. 532-562. MR 2002f: 11125

4. M. D. Coleman, The distribution of points at which binary quadratic forms are prime, Proc. London Math. Soc. (3) 61 (1990), 433-456. MR 91j:11077 
5. __ A zero-free region for the Hecke L-functions, Mathematika 37 (1990), 287-304. MR 92c:11131

6. (1993), 53-83. MR 94h:11086

7. 1 , Relative norms of prime ideals in small regions, Mathematika 43 (1996), 40-62. MR 97f:11094

8. G. Harman, On the distribution of $\alpha p$ modulo one, J. London Math. Soc. (2) 27 (1983), 9-18. MR 84d:10044

9. - On the distribution of $\alpha p$ modulo one II, Proc. London Math. Soc. (3) 72 (1996), 241-260. MR 96k:11089

10. G. Harman and P. A. Lewis, Gaussian primes in narrow sectors, Mathematika, to appear.

11. D. R. Heath-Brown, The number of primes in a short interval, J. Reine Angew. Math. 389 (1988), 22-63. MR 89i:11099

12. D. R. Heath-Brown and H. Iwaniec, On the difference between consecutive primes, Invent. Math. 55 (1979), 49-69. MR 81h:10064

13. M. N. Huxley, On the difference between consecutive primes, Invent. Math. 15 (1972), 164170. MR 45:1856

14. H. L. Montgomery, Topics in Multiplicative Number Theory, Lecture Notes in Math. 227, Springer-Verlag, Berlin-New York, 1971. MR 49:2616

15. W. Narkiewicz, Elementary and Analytic Theory of Algebraic Numbers, Monografie Matematyczne 57, PWN-Polish Scientific Publishers, Warsaw, 1974. MR 50:268

16. H. Rademacher, On the Phragmén-Lindelöf theorem and some applications, Math. Z. 72 (1959/60), 192-204. MR 22:7982

17. E. C. Titchmarsh, The Theory of the Riemann Zeta-Function, Second ed., edited and with a preface by D. R. Heath-Brown, The Clarendeon Press, Oxford University Press, New York, 1986. MR 88c: 11049

18. N. Watt, Kloosterman sums and a mean value for Dirichlet polynomials, J. Number Theory 53 (1995), 179-210. MR 96f:11109

Department of Mathematics, Royal Holloway University of London, Egham, Surrey TW20 0EX, United Kingdom

E-mail address: G.Harman@rhul.ac.uk

Department of Mathematics, University of Toronto, Toronto, Ontario, Canada M5S $3 \mathrm{G} 3$

E-mail address: kumchev@math.toronto.edu

School of Mathematics, Cardiff University, P.O. Box 926, Cardiff CF24 4YH, Wales, United KingDOM

E-mail address: LewisPA3@Cardiff.ac.uk 